\title{
CONTRIBUIÇÕES DA BIOLOGIA MOLECULAR PARA CARACTERIZAÇÃO, CONSERVAÇÃO E USO DO GÊNERO Croton L. (Euphorbiaceae)
}

Thalana Souza Santos Silva ; Janaína Silva Freitas²; Elisa Susilene Lisboa dos Santos $^{2}$; Carlos Bernard Moreno Cerqueira-Silva ${ }^{2 *}$

1Mestre em Ciências Ambientais e Doutoranda pelo Programa Multicêntrico de Pósgraduação em Bioquímica e Biologia Molecular, Universidade Estadual do Sudoeste da Bahia, Itapetinga, Bahia, Brasil.

2Docentes do Departamento de Ciências Exatas e Naturais da Universidade Estadual do Sudoeste da Bahia, Itapetinga, Bahia, Brasil.

*Autor para correspondência: csilva@uesb.edu.br

\section{Recebido em: 06/04/2019 - Aprovado em: 10/06/2019 - Publicado em: 30/06/2019 DOI: 10.18677/EnciBio_2019A40}

\begin{abstract}
RESUMO
O conhecimento genético-molecular das espécies que apresentam relevância enquanto recurso natural é fundamental para elaboração de estratégias de conservação e uso sustentável da biodiversidade. Os marcadores moleculares são importantes ferramentas para obtenção de dados genéticos relativos desde as estimativas populacionais até identificação de regiões genômicas associados a características fenotípicas. Assim, objetivou-se apresentar um panorama do conhecimento disponível na literatura sobre a aplicação de marcadores moleculares para o estudo de espécies do gênero Croton, indicando tanto as principais lacunas, como as perspectivas em relação às ferramentas moleculares disponíveis e sua aplicação na compreensão do gênero, tendo como fim a conservação e uso dessas espécies. Para tanto, foram realizadas buscas em bases de dados (indexadores) de publicações científicas. Em síntese, os estudos genéticos com a aplicação de marcadores moleculares restringiram-se a sete espécies de Croton spp., o que corresponde a menos de $1 \%$ das espécies do gênero, e em sua maioria foram utilizados marcadores moleculares dominantes, fato que limita a possibilidade dos autores em ampliar suas discussões e conclusões. Os dados apresentados indicam a escassez de conhecimento relativo as características genéticas e sobre a estrutura genética populacional para as espécies de Croton spp.. Consequentemente, essa realidade atesta um conjunto de oportunidades para realização de novas pesquisas que contribuam para caracterização e uso dessa biodiversidade.
\end{abstract}

PALAVRAS-CHAVE: diversidade genética, marcadores moleculares, recursos genéticos vegetais 


\title{
CONTRIBUTIONS OF MOLECULAR BIOLOGY FOR CHARACTERIZATION, CONSERVATION AND USE OF THE GENUS Croton L. (Euphorbiaceae)
}

\begin{abstract}
The genetic-molecular knowledge of the species that present relevance as a natural resource is fundamental for the elaboration of strategies of conservation and sustainable use of biodiversity. Molecular markers are important tools for obtaining relative genetic data from population estimates to identification of genomic regions associated with phenotypic characteristics. The aim of this study was to present an overview of the available knowledge in the literature on the application of molecular markers for the study of species of the genus Croton, indicating both the main gaps and perspectives regarding the available molecular tools and its application in the understanding of the genus, aiming at the conservation and use of these species. For that, searches were made on databases (indexers) of scientific publications. In summary, genetic studies with the application of molecular markers were restricted to seven species of Croton spp., corresponding to less than $1 \%$ of the species of the genus, and most dominant molecular markers were used, a fact that limits the possibility of authors to broaden their discussions and conclusions. The data presented indicate the lack of knowledge regarding the genetic characteristics and the population genetic structure for Croton spp.. Consequently, this reality testifies to a set of opportunities for conducting new research that contributes to the characterization and use of this biodiversity.
\end{abstract}

KEYWORDS: genetic diversity, molecular markers, plant genetic resources

\section{INTRODUÇÃO}

O gênero Croton L. é o segundo maior gênero da família Euphorbiaceae, com aproximadamente 1.200 espécies. As espécies do gênero apresentam ampla distribuição mundial com a maioria dos seus representantes distribuídos nas regiões tropicais, predominantemente no continente Americano, sendo o Brasil (300 espécies), Madagascar (150 espécies), Caribe (150 espécies) e México (100 espécies) considerados os mais importantes centros de diversidade do gênero (BERRY et al., 2005). No Brasil, as espécies de Croton estão amplamente distribuídas em diversos ambientes e vegetações, havendo uma maior concentração de espécies nas regiões Sudeste (149 espécies) e Nordeste (115 espécies) do país (CORDEIRO et al., 2019).

A distribuição geográfica das espécies de Croton em diferentes paisagens é resultado de uma soma de fatores que envolvem interações ecológicas estabelecidas pelas espécies do gênero, ressaltando-se os eventos de polinização e dispersão, relacionados com o sucesso reprodutivo, bem como as estratégias de defesa em resposta à ação dos predadores e aos fatores limitantes do ambiente em que essas espécies ocorrem.

Além da importância ecológica, as espécies do gênero Croton constituem um importante recurso natural, sendo utilizadas para diversas finalidades, com destaque para o uso medicinal que tem despertado o interesse em potencial para descoberta de medicamentos. Estudos têm demonstrado uma riqueza na composição química das espécies de Croton, com a identificação de cerca de 400 compostos químicos, dos quais muitos apresentam atividade biológica comprovada (WEN-HUI et al., 2018), a exemplo das atividades citotóxica [Croton heliotropiifolius Kunth (ALENCAR et al., 2016)]; antiangiogênica [Croton crassifolius 
Geiseler. (LIANG et al., 2017)]; anti-inflamatória [Croton urucurana Baill. (CORDEIRO et al., 2016)]; inseticida [Croton argyrophyllus Kunth. (CRUZ et al., 2015; CRUZ et al., 2017)]; antinociceptivo [Croton cordiifolius Baill. (NOGUEIRA et al., 2015)], fato que naturalmente agrega valor econômico em potencial para muitas espécies. Dentre essas espécies muitas são nativas do Brasil (a exemplo de Croton heliotropiifolius, C. bonplandianum Bail, C. urucurana, C. muscicarpa Müll. Arg., C. argyrophylloides Muell. Arg. e C. limae A.P. Gomes, M.F. Sales P.E. Berry).

Contudo a extração insustentável desse recurso natural, aliado ao elevado número de espécies de Croton endêmicas do Brasil (aproximadamente 250) (CORDEIRO et al., 2019), implica em um cenário preocupante para a preservação das espécies do gênero no país, tendo em vista que aproximadamente 39\% dessas espécies encontram-se sob ameaça (POLLITO et al., 2004). Este fato demonstra a urgência na aplicação de medidas de conservação, tais como, a proteção dos ambientes de ocorrência dessas espécies por meio da criação e ampliação de áreas protegidas (in situ) (POLLITO et al., 2004), bem como, estratégias de conservação ex situ, a exemplo dos bancos ativos de germoplasma e coleções.

No contexto apresentando, posto as potencialidades do gênero e os riscos inerentes a sua conservação, torna-se evidente a demanda por estudos de caracterização dessa biodiversidade, visto que somente com efetivo conhecimento da biodiversidade (especialmente quanto a sua variabilidade e estruturação genética) tornar-se viável o avanço no estabelecimento e na consolidação de estratégias de conservação e uso dos recurso naturais (NASS, 2011; CERQUEIRASILVA et al., 2014).

Técnicas da genética e biologia molecular, a exemplo dos marcadores moleculares, fornecem informações úteis em diferentes estudos e etapas metodológicas, que incluem desde a coleta, caracterização até o efetivo uso dos recursos genéticos vegetais, a exemplo dos programas de melhoramento de plantas (FALEIRO et al., 2018). Os marcadores moleculares, em complemento a outras técnicas utilizadas para a caracterização dos recursos genéticos, como os marcadores morfológicos e bioquímicos, apresentam vantagens, pois permitem a avaliação diretamente ao nível de DNA, livre de influências do ambiente ou do estágio de desenvolvimento do organismo analisado, além de possibilitar a obtenção de um número praticamente ilimitado de polimorfismos genéticos (FALEIRO et al., 2018).

Diante da gama de marcadores moleculares, diversos fatores podem influenciar na escolha da técnica mais adequada aos objetivos do estudo e ao organismo alvo. Os marcadores moleculares diferem entre si quanto ao nível de polimorfismo detectado, a abundância no genoma, a especificidade de locus, a reprodutibilidade e os requisitos de detecção e automação da técnica. Também devem ser considerados na determinação dos marcadores a serem empregados em um estudo, os fatores relativos a infraestrutura disponível, aos requisitos técnicos e de custos e o nível de conhecimento genético da espécie a ser caracterizada no estudo (MONDINI et al., 2009; HAYWARD et al., 2015; TURCHETTO-ZOLET et al., 2017; FALEIRO et al., 2018).

Tendo em vista que os marcadores moleculares, têm sido empregados para identificação, caracterização e avaliação dos recursos genéticos, e que as informações geradas por essas ferramentas são importantes para a implementação de estratégias de conservação e uso das espécies, objetivou-se apresentar um panorama do conhecimento relativo à aplicação dos 
marcadores moleculares para espécies do gênero Croton, indicando tanto as principais lacunas como as perspectivas em relação às ferramentas moleculares disponíveis e sua aplicação nas estimativas genéticas do gênero, tendo como fim a conservação e uso dessas espécies.

\section{MATERIAL E MÉTODOS}

Foi realizada uma revisão integrativa com base em buscas realizadas em diversas bases de dados de publicações científicas: Pubmed (https://www.ncbi.nlm.nih.gov/pubmed), Doaj (https://doaj.org/), Google scholar (https://scholar.google.com.br/), semantic scholar (https://www.semanticscholar.org/), SciELO (http://www.scielo.org/php/index.php), Web of Science (https://login.webofknowledge.com) e SCOPUS (https://www.scopus.com). Os termos utilizados em português e inglês na pesquisa foram Croton, em combinação com os termos "genética" ou "genetic", e "marcador molecular" ou "molecular markers".

Quanto aos critérios de inclusão, foram considerados os artigos originais disponíveis eletronicamente, em português, inglês e/ou espanhol. Não foi estabelecido um período específico para ocorrência das publicações, possibilitando assim uma abrangência quantitativa de publicações que efetivamente representam o estado da arte relativa a temática considerada neste estudo. De posse dos artigos, foi realizada sequencialmente leitura exploratória com vistas a identificação dos artigos que apresentassem como objetivo a aplicação de marcadores moleculares em estudo de espécies do gênero Croton e posteriormente uma leitura crítica dos artigos selecionados.

\section{RESULTADOS E DISCUSSÃO}

Apesar dos avanços obtidos nas últimas décadas no desenvolvimento dos marcadores moleculares, e a sua aplicação em estudos genéticos de diversas espécies vegetais, sobretudo em espécies cultivadas comercialmente, o mesmo progresso não é observado para as espécies do gênero Croton (Figura 1), com média de intervalo entre o desenvolvimento inicial das técnicas e sua efetiva aplicação no gênero Croton variando de 15 a 22 anos, além de limitado a menos de 10 espécies. A despeito dessa realidade, o advento da técnica da reação em cadeia da polimerase (PCR - Polymerase Chain Reaction) no início dos anos 1980 impulsionou o desenvolvimento da maioria dos marcadores disponíveis, como AFLP (Amplified Fragment Length Polymorphism), SSR (Simple Sequence Repeats), ISSR (Inter Simple Sequence Repeat), RAPD (Random Amplified Polymorphic DNA), RGA (Resistance genes analogs) (Figura 1A), entre outros. Desde então, os avanços nas técnicas de sequenciamento em larga escala tem levado ao desenvolvimento e aprimoramento dos marcadores moleculares, como os SNPs (Single Nucleotide Polymorphism) (TURCHETTO-ZOLET et al., 2017; NADEEM et al., 2018). 
(A)

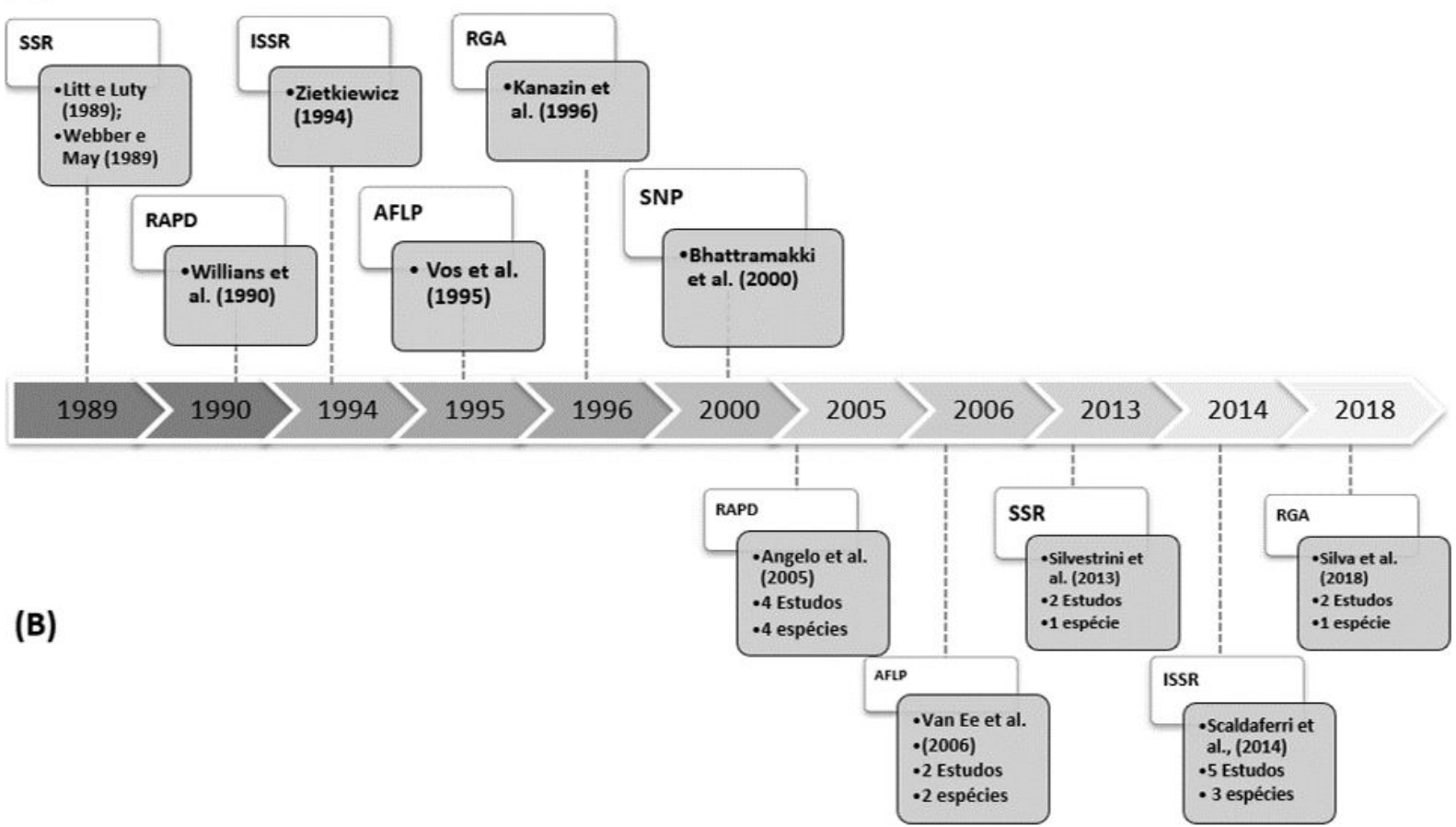

FIGURA 1. Esquema ilustrativo dos principais marcadores moleculares e suas referências iniciais $(\mathbf{A})$, bem como os estudos publicados utilizando marcadores moleculares no gênero Croton, a sua referência inicial, e o quantitativo de estudos e espécies (B). Fonte: autoria própria.

Estudos acerca da caracterização genética de Croton com a aplicação de marcadores moleculares estão disponíveis para sete espécies (Croton cajucara Benth., Croton alabamensis E.A. Sm. ex Chapm., Croton floribundus Spreng., Croton linearifolius Mull. Arg., Croton heliotropiifolius Kunth., Croton tetradenius baill e Croton antisyphiliticus Mart. ex M. Arg.), o que corresponde a menos de $1 \%$ das espécies do gênero (quadro 1), atestando a efetiva carência de dados e a necessidade de proposições relacionadas ao entendimento da diversidade e estrutura genética do gênero. Além do que, a escassez de resultados genéticomoleculares implica em ausência de informações relativas a identificação de regiões genômicas associadas a expressão de características de interesse para adaptação e uso das espécies. A aplicação de marcadores moleculares para as espécies do gênero iniciou-se em 2005, com a proposição de um estudo de diversidade de $C$. cajucara com base no marcador RAPD (ANGELO et al., 2005) (Figura 1B). A maioria dos estudos (80\%) é baseada no uso de marcadores moleculares dominantes (quadro 1), que em função do seu padrão de segregação, apresenta limitações quanto a realização de algumas estimativas genéticas, sobretudo quanto associadas a questões populacionais. $O$ emprego de marcadores co-dominantes como os SSR, iniciou-se em 2013 com o desenvolvimento de primers e estimativas de diversidade e estrutura genética para a espécie C. floribundus (SILVESTRINI et al., 2013; SILVESTRINI et al., 2015). O avanço no uso desses marcadores, em diferentes espécies e populações de Croton spp. deverá permitir uma ENCICLOPÉDIA BIOSFERA, Centro Científico Conhecer - Goiânia, v.16 n.29; p.543 
discussão mais efetiva quanto a variabilidade e estruturação dessa biodiversidade.

QUADRO 1. Apresentação descritiva e quantitativa de estudos publicados utilizando diferentes marcadores moleculares para o gênero Croton.

\begin{tabular}{|c|c|c|c|c|}
\hline $\begin{array}{l}\text { Marcadores } \\
\text { moleculares }\end{array}$ & $\begin{array}{l}\text { Padrão de } \\
\text { herança }\end{array}$ & $\begin{array}{c}\text { Objetivo dos } \\
\text { estudos }\end{array}$ & $\begin{array}{l}\text { Espécies } \\
\text { avaliadas }\end{array}$ & Referências \\
\hline \multirow{4}{*}{ RAPD } & \multirow{4}{*}{ Dominante } & \multirow{4}{*}{$\begin{array}{l}\text { Estimativas } \\
\text { de } \\
\text { diversidade } \\
\text { e estrutura } \\
\text { genética; } \\
\text { - Comparação } \\
\text { de métodos } \\
\text { para } \\
\text { estimativas } \\
\text { de } \\
\text { diversidade } \\
\text { genética. }\end{array}$} & C. cajucara & $\begin{array}{l}\text { ANGELO et al., } \\
2005\end{array}$ \\
\hline & & & C. floribundus & $\begin{array}{c}\text { SOUZA et al., } \\
2012\end{array}$ \\
\hline & & & C. linearifolius & $\begin{array}{l}\text { SCALDAFERRI } \\
\text { et al., } 2014\end{array}$ \\
\hline & & & $\begin{array}{c}\text { C. } \\
\text { heliotropiifolius }\end{array}$ & $\begin{array}{c}\text { ROCHA et al., } \\
2016\end{array}$ \\
\hline \multirow{5}{*}{ ISSR } & \multirow{5}{*}{ Dominante } & \multirow{5}{*}{$\begin{array}{l}\text { - Estimativas } \\
\text { de } \\
\text { diversidade } \\
\text { e estrutura } \\
\text { genética; } \\
\text { - Comparação } \\
\text { de métodos } \\
\text { para } \\
\text { estimativas } \\
\text { de } \\
\text { diversidade } \\
\text { genética; } \\
\text { - Seleção de } \\
\text { primers. }\end{array}$} & C. linearifolius & $\begin{array}{l}\text { SCALDAFERRI } \\
\text { et al., } 2014\end{array}$ \\
\hline & & & $\begin{array}{c}C . \\
\text { heliotropiifolius }\end{array}$ & $\begin{array}{c}\text { ROCHA et al., } \\
2016\end{array}$ \\
\hline & & & C. tetradenius & $\begin{array}{c}\text { ALMEIDA- } \\
\text { PEREIRA et al., } \\
2017\end{array}$ \\
\hline & & & C. linearifolius & SILVA et al.,2018 \\
\hline & & & C. linearifolius & $\begin{array}{l}\text { SILVA et al.,no } \\
\text { prelo }\end{array}$ \\
\hline \multirow[b]{2}{*}{ AFLP } & \multirow[b]{2}{*}{ Dominante } & \multirow{2}{*}{$\begin{array}{l}\text { Filogenia; } \\
\text { Estimativas } \\
\text { de } \\
\text { diversidade } \\
\text { genética. }\end{array}$} & $\begin{array}{c}\text { C. } \\
\text { alabamensis }\end{array}$ & $\begin{array}{c}\text { VAN EE et al., } \\
2006\end{array}$ \\
\hline & & & $\begin{array}{c}\text { C. } \\
\text { antisyphiliticus }\end{array}$ & $\begin{array}{l}\text { OLIVEIRA et } \\
\text { al.,2016 }\end{array}$ \\
\hline \multirow[b]{2}{*}{ RGA } & & \multirow{2}{*}{$\begin{array}{l}\text { - Estimativas } \\
\text { de } \\
\text { diversidade } \\
\text { e estrutura } \\
\text { genética; } \\
\text { - Seleção de } \\
\text { primers. }\end{array}$} & C. linearifolius & SILVA et al.,2018 \\
\hline & & & C. linearifolius & $\begin{array}{l}\text { SILVA et al.,(no } \\
\text { prelo) }\end{array}$ \\
\hline
\end{tabular}




\begin{tabular}{|c|c|c|c|c|}
\hline & & $\begin{array}{l}\text { Estimativas } \\
\text { de } \\
\text { diversidade }\end{array}$ & C. floribundus & $\begin{array}{c}\text { SILVESTRINI et } \\
\text { al., } 2013\end{array}$ \\
\hline SSR & Codominante & $\begin{array}{l}\text { e estrutura } \\
\text { genética; } \\
\text { - Desenvolvim } \\
\text { ento de } \\
\text { primers. }\end{array}$ & C. floribundus & $\begin{array}{l}\text { SILVESTRINI et } \\
\text { al., } 2015\end{array}$ \\
\hline
\end{tabular}

Os marcadores RAPD e ISSR foram os mais utilizados em Croton totalizando quatro e cinco estudos, respectivamente (quadro 1 e Figura 1B). Dentre as vantagens que podem justificar a escolha por essas técnicas, destacam-se a simplicidade, rapidez na obtenção de dados, baixo custo, demanda de quantidades mínimas de DNA para realização das análises e a possibilidade de estudar espécies sem informações prévias de sequências de DNA para construção dos primers (TURCHETTO-ZOLET et al., 2017). Tais características são especialmente úteis para espécies pouco estudadas e com interesse comercial pouco explorado, situação da maioria das espécies do gênero Croton. Apesar das limitações inerentes ao uso dos marcadores dominantes, como a impossibilidade de diferenciar os locos em heterozigose, a aplicação desses marcadores geram informações que podem contribuir para ampliação do conhecimento genético de Croton.

Em estudo com C. cajucara, espécie que apresenta potencial econômico na indústria de cosméticos, o marcador RAPD foi aplicado para estimar a diversidade genética entre 16 acessos de dois morfotipos da espécie (ANGELO et al., 2006). Marcadores do tipo RAPD também foram utilizados para analisar a estrutura genética da espécie $C$. floribundus em diferentes pontos de coleta a fim de estabelecer estratégias para a coleta de sementes usadas em reflorestamentos da Floresta Atlântica (SOUZA et al., 2012). Esses dois estudos realizados por Angelo et al. (2006) e Souza et al. (2012), atestam a importância dos marcadores moleculares como subsídios para fortalecer ações de conservação e/ou (pré)melhoramento.

O RAPD também foi utilizado em conjunto com marcadores ISSR para comparar métodos para estimativas de diversidade genética em $C$. linearifolius (SCALDAFERRI et al., 2014) e estimar a variabilidade genética em $C$. heliotropiifolius (ROCHA et al., 2016). Estas espécies têm despertado o interesse dos pesquisadores por apresentarem potencial inseticida e medicinal, respectivamente. Os marcadores ISSR apresentam vantagens quando comparados ao RAPD, pois possuem alta reprodutibilidade devido ao uso de sequências mais longas (aproximadamente 20 pb) na composição dos iniciadores e por possuírem, consequentemente maiores temperaturas de anelamento (MONDINI et al., 2009; IBRAHIM et al., 2010).

O marcador ISSR foi também utilizado para analisar a diversidade genética entre 40 indivíduos de $C$. tetradenius presentes em quatro populações naturais no Estado de Sergipe, Brasil (ALMEIDA-PEREIRA et al., 2017) contribuindo para o avanço do entendimento genético populacional da espécie em seu ambiente natural. Por sua vez, Silva et al. (2018) selecionaram 16 iniciadores ISSR e 14 combinações de iniciadores RGA, tendo por critérios a ENCICLOPÉDIA BIOSFERA, Centro Científico Conhecer - Goiânia, v.16 n.29; p.545 
resolução das marcas e os padrões polimórficos, a fim de subsidiar estudos genéticos em $C$. linearifolius. Os marcadores ISSR e RGA selecionados por Silva et al. (2018) foram empregados para estimar a estrutura e diversidade genética em 61 indivíduos de C. linearifolius presentes na Floresta Nacional Contendas do Sincorá (FLONA) (SILVA et al., no prelo). Estudos dessa natureza, como os de Silva et al. (2018), demonstram a possibilidade de uso conjunto de diferentes marcadores moleculares em estudos genéticos. Tal estratégia pode contribuir para uma maior amostragem do genoma estudado.

A técnica AFLP foi empregada para avaliar a variabilidade genética em populações naturais de $C$. antisyphiliticus, espécie utilizada no tratamento de infecções do aparelho urogenital, presentes nos estados de Minas Gerais, São Paulo e Goiás (OLIVEIRA et al., 2016). Além das características inerentes aos marcadores dominantes, os marcadores AFLP apresentam como desvantagens a exigência de uma maior quantidade de DNA, várias etapas e reagentes que aumentam o custo da técnica (TURCHETTO-ZOLET et al., 2017), sendo estes fatores potenciais/prováveis motivos para o reduzido número de estudos de Croton com estes marcadores, se comparado com os RAPD e ISSR (quadro 1).

Marcadores moleculares AFLP foram empregados em conjunto com dados de sequências de DNA para estimar a diversidade genética e para reconstrução das relações filogenéticas em $C$. Alabamensis com ocorrência nos estados do Texas e Alabama nos Estados Unidos (VAN EE et al., 2006). O uso de marcadores AFLP tem sido utilizado com êxito em estudos que visam elucidar as relações filogenéticas entre espécies relacionadas e táxons infraespecíficos, não sendo indicada a sua aplicação para comparações entre organismos filogeneticamente distantes devido a alta variabilidade apresentada por esse marcador (TURCHETTO-ZOLET et al., 2017).

Fatores como o elevado número de espécies, somados a ampla distribuição geográfica e considerável diversidade morfológica, contribuem para que o gênero Croton seja considerado de alta complexidade taxonômica, sendo estimulada a realização de estudos para elucidação das relações filogenéticas do gênero (RIINA et al., 2009). Estratégias de estudos moleculares associadas a identificação, sequenciamento e comparação de regiões conservadas do genoma (a exemplo da técnica de DNA Barcode) podem contribuir na identificação e diferenciação de espécies de Croton, auxiliando no desenvolvimento de estudos taxonômicos e filogenéticos (OSATHANUNKUL et al., 2015).

Um novo arranjo para as seções do gênero Croton foi proposto por Berry et al. (2005) por meio de estudos filogenéticos moleculares tendo como base a análise de dados moleculares das regiões ITS, do DNA nuclear ribossômico, e do fragmento trnL-F, do DNA plastidial. Após esse trabalho inicial, outras análises filogenéticas moleculares têm contribuído para a construção de uma classificação infragenérica de Croton (RIINA et al., 2009; RIINA et al., 2010; CARUZO et al., 2011; VAN EE et al., 2011; VAN EE; BERRY, 2011; RIINA et al., 2014). Como resultado do aumento no número de estudos taxonômicos e filogenéticos, o conhecimento acerca de Croton vem crescendo substancialmente. Apesar dos avanços, as relações dentro de muitas seções ainda não foram elucidadas (CARUZO et al., 2011).

Os marcadores co-dominantes SSR combinados com análises citogenéticas, foram empregados por Silvestrini et al. (2013) para investigar as implicações do uso dessa classe de marcadores no estudo de $C$. floribundus, uma espécie poliplóide. Dos 16 primers testados, sete locos foram úteis, 
gerando um total de 69 alelos, com número médio de 9,9 alelos por loco. Os autores confirmaram a eficiência desse marcador para estudos genético de populações de espécies pioneiras poliplóides.

Os marcadores SSR também foram utilizados por Silvestrini et al. (2015) para investigar como os processos ecológicos e os fatores genéticos associados aos distúrbios naturais ou antrópicos afetam a diversidade e estrutura genética de populações de $C$. floribundus em diferentes estágios sucessionais. Foram obtidos 77 alelos com o uso de sete locos de SSR em 225 indivíduos de C. floribundus. A combinação de análises obtidas com os marcadores moleculares nucleares SSR e cloroplastidias sugeriram que o fluxo gênico por pólen é responsável por manter a diversidade genética dentro das populações de C. floribundus, tanto na floresta primária quanto na floresta secundária, em estágio inicial de sucessão.

Os marcadores SSR são os mais indicados para estudos populacionais, pois são co-dominantes, possibilitando a identificação de heterozigotos, além de apresentarem alto polimorfismo e serem abundantes nos genomas das plantas. Apesar das vantagens apresentadas, a grande limitação do uso dessa técnica em larga escala, consiste no desenvolvimento de primers específicos para espécie a ser analisada, o que pode demandar maior custo e tempo (FERREIRA et al., 2018). A despeito dessas limitações técnicas, é natural que a semelhança do que se observa para a grande maioria das espécies vegetais, se observe um crescimento no número de estudos genéticos baseados em marcadores codominantes no gênero Croton.

$\mathrm{Na}$ maioria dos estudos foi estimada a diversidade genética (Tabela 1) de Croton (quadro 1), e em geral foram encontrados altos níveis de diversidade genética dentro das populações das espécies estudadas, sendo detectado algum nível de estruturação genética na maioria dos estudos. O nível de variabilidade genética implica no potencial evolutivo de uma espécie, e determina em grande parte, suas chances de sobrevivência frente às flutuações ambientais provocadas pelos fatores bióticos e abióticos (FRANKHAM, 2003). O conhecimento sobre a estruturação genética de populações de plantas pode fornecer informações importantes para o desenvolvimento de estratégias de conservação ex situ e in situ, e para o manejo dos recursos vegetais (CERQUEIRA-SILVA et al., 2014).

TABELA 1- Descrição dos estudos publicados com a aplicação de marcadores moleculares para o gênero Croton, cujo objetivo foi a estimativa de estrutura e diversidade genética.

\begin{tabular}{cccc}
\hline $\begin{array}{c}\text { Marcador } \\
\text { molecular }\end{array}$ & Espécie & $\begin{array}{c}\text { Estruturação } \\
\text { genética (Amova) }\end{array}$ & Polimorfismo (\%) \\
\hline RAPD & C. floribundus (A) & Baixa & 94.9 \\
RAPD & C. cajucara (B) & -- & 82.3 \\
AFLP & C. antisyphiliticus (A) & Alta & 92.1 \\
AFLP & C. alabamensis (A) & Moderada & 100 \\
ISSR & C. tetradenius (A) & Baixa & 94,8 \\
ISSR e RGA & C. linearifolius (A) & Moderada & 81,3 \\
ISSR e RAPD & C. heliotropiifolius (A) & -- & 95.6 \\
SSR & C. floribundus (A) & Moderada/Alta & 100 \\
\hline
\end{tabular}

A- população natural; B- banco de germoplasma; Amova- Análise Molecular de Variância; -- estudos que não realizaram a análise Amova. 
Na maioria dos estudos com aplicação de marcadores moleculares em Croton foram investigadas amostras coletadas em fragmentos de populações naturais (Tabela 1), demonstrando que a maior parte das pesquisas tem relação com estudos relativos a conservação, do que necessariamente com o melhoramento. Três das espécies estudadas estão presentes em unidades de conservação (C. floribundus, C. helitropifolius e C. linearifolius), e apenas uma espécie dentre as sete investigadas com marcadores moleculares (C. cajucara) está mantida em banco de germoplasma. Além disso, do total de espécies do gênero investigadas duas são endêmicas do Brasil (C. floribundus, $C$. linearifolius). O panorama apresentado implica em uma importante lacuna no conhecimento da biodiversidade inerente ao gênero Croton e consequentemente sobre o status de conservação das espécies do gênero, já que os dados gerados por meio da utilização das técnicas moleculares podem elucidar o nível de diversidade genética, possibilitando a implementação de estratégias de conservação e uso sustentável dessas espécies.

A escassez de estudos sobre Croton, aliado ao elevado número de espécies endêmicas no Brasil, país no qual foram encontrados a maior parte dos estudos, demonstram a urgência na aplicação de medidas de proteção das áreas de ocorrência dessas espécies no país, bem como o estabelecimento de estratégias de fomento que potencialize a caracterização dessa biodiversidade com fins de conservação e uso.

\section{CONCLUSÃO}

Os dados apresentados indicam a escassez de conhecimento relativo as características genéticas e sobre a variabilidade e a estrutura genética populacional para as espécies de Croton spp.. Consequentemente, essa realidade atesta um conjunto de oportunidades para realização de novas pesquisas, visto que os estudos genéticos com a aplicação de marcadores moleculares restringiram-se a menos de $1 \%$ das espécies de Croton, e em sua maioria decorrem exclusivamente do uso de marcadores moleculares dominantes, fato que dentre outras implicações, limita a possibilidade dos autores em ampliar suas discussões e conclusões.

Embora relevante para as atividades de conservação e melhoramento genético há uma escassez de estudos genético-populacionais com o uso de técnicas moleculares mais robustas, a exemplo dos marcadores co-dominantes, tendo em vista que os estudos com esta classe de marcadores limitaram-se a investigação de uma única espécie, $C$. floribundus. Dessa forma, vislumbra-se um necessário e natural crescimento na aplicação de marcadores mais informativos como os SSR e os baseados em sequências, a exemplo dos SNPs (Single Nucleotide Polymorphism).

\section{AGRADECIMENTOS}

Ao Programa de Pós-Graduação em Ciências Ambientais (PPGCA/UESB) e à Coordenação de Aperfeiçoamento de Pessoal de Nível Superior (CAPES) pela bolsa de Mestrado concedido a Silva, T.S.S., oportunidade na qual foi possível a autora realizar seus estudos com o gênero Croton. 


\section{REFERÊNCIAS}

ALMEIDA-PEREIRA, C. S.; SILVA, A. V. C.; ALVES, R. P.; FEITOSA-ALCANTARA, R. B.; ARRIGONI-BLANK, M. F. et al. Genetic diversity of native populations of Croton tetradenius Baill. using ISSR markers. Genetic Molecular Research, v. 16, n. 2, $\quad$ p. 1-12, 2017. Disponível em: $<$ https://www.alice.cnptia.embrapa.br/alice/bitstream/doc/1076428/1/Artigo5Crotontet radeniusGMR.pdf>. doi: http://dx.doi.org/10.4238/gmr16029602.

ALENCAR, G. O.; CALIXTO, JÚNIOR J. T.; BOLIGON, A. A.; ATHAYDE, M. L.; BEZERRA, C. A. et al. Atividade antiparasitária e citotóxica do extrato etanólico de folhas de Croton heliotropiifolius Kunth (Euphorbiaceae) Rev. Bras. PI. Med., São Paulo, v.18, n.4, p.797-803, 2016. Disponível em: https://www.researchgate.net/publication/320387269_Atividade_antiparasitaria_e_cit otoxica_do_extrato_etanolico_de_folhas_de_Croton_heliotropiifolius_Kunth_Euphor biaceae doi: $10.1590 / 1983-084 X / 0039$.

ANGELO, P. C. S.; CHAVES, F. C. M.; BIZZO, H. R.; XAVIER, J. J. B. N.; CRUZ, J. C. et al. Genetic diversity in sacaca (Croton cajucara Benth.) accessed by RAPD markers. Revista Brasileira de Plantas Medicinais, v. 8, n.esp., p.18-22, 2006. Disponível em: <https://www.alice.cnptia.embrapa.br/bitstream/doc/680888/1/8esp1822.pdf>.

BERRY, P. E.; HIPP, A. L.; WURDACK, K. J.; EE, B. V.; RIINA, R. Molecular phylogenetics of the giant genus Croton and tribe Crotoneae (Euphorbiaceae sensu stricto) using ITS and trnL-trnF DNA sequence data. American Journal of Botany, v. 92, n.9, p.1520-1534, $2005 . \quad$ Disponível em: $<$ https://pdfs.semanticscholar.org/6889/216a035a04ef54b59d51a478734aae8c8e51. pdf>.

CARUZO, M. B. R.; VAN EE, B.; CORDEIRO, I.; BERRY, P. E.; RIINA, R. Molecular phylogenetics and character evolution of the "sacaca" clade: novel relationships of Croton section Cleodora (Euphorbiaceae). Molecular Phylogenetics and Evolution, v.60, p.193-206, 2011. doi: https://doi.org/10.1016/j.ympev.2011.04.013

CERQUEIRA-SILVA, C. B. M.; NUNES, O.; SANTOS, E. S. L.; CORRÊA, R. X.; SOUZA, A. P. Genetic breeding and diversity of the genus Passiflora: progress and perspectives in molecular and genetic studies. International Journal of Molecular Sciences, v. 15, n. 8, p. 14122-14152, 2014. Disponível em: $<$ https://pdfs.semanticscholar.org/2891/eaffd466a8aca516e81ddb0daf46d89cab23.p df?_ga $=2.266437134 .166968736 .1554424627-40333914.1552833034>. \quad$ doi: https://doi.org/10.3390/ijms150814122.

CORDEIRO, I.; SECCO, R.; CARNEIRO-TORRES, D. S.; LIMA, L. R. DE; CARUZO, M. B. R. et al. Croton. In: Flora do Brasil 2020 em construção. Jardim Botânico do Rio de Janeiro. Disponível em: $<$ http:/floradobrasil.jbrj.gov.br/reflora/floradobrasil/FB17497>. Acesso em: 17.02. 2019.

CORDEIRO, K. W.; FELIPE, J. L.; MALANGE, K. F.; DO PRADO, P. R.; DE OLIVEIRA, F. P. et al. Anti-inflammatory and 
antinociceptive activities of Croton urucurana Baillon bark. Journal of Ethnopharmacology, v.183, n.13, p.128-135, $2016 . \quad$ doi: https://doi.org/10.1016/j.jep.2016.02.051

CRUZ, R. C. D.; SILVA, S. L. C. E; SOUZA, I. A.; GUALBERTO, S. A.; CARVALHO, K. S. et aL. Toxicological Evaluation of Essential Oil From the Leaves of Croton argyrophyllus (Euphorbiaceae) on Aedes aegypti (Diptera: Culicidae) and Mus musculus (Rodentia: Muridae). J Med Entomol. v.54, n.4, p.985-993, 2017. doi: https://doi.org/10.1093/jme/tjw239

CRUZ, R. C. D.; CARVALHO, K. S.; SILVA, S. L. C. E.; GUAlBeRTO, A. S. Avaliação da atividade larvicida dos extratos aquosos e do hidrolato obtido das folhas de Croton argyrophyllus sobre o Aedes aegypti. Enciclopédia Biosfera, v.11, n. 21, p.2835-2841, 2015. Disponível em: http://www.conhecer.org.br/enciclop/2015b/multidisciplinar/avaliacao\%20da\%20ativid ade\%20larvicida.pdf

FALEIRO, F. G.; AMABILE, R. F.; CERQUEIRA-SILVA, C. B. M. Marcadores moleculares aplicados ao melhoramento genético de plantas. In: AMABILE, R.F.; VILELA, M. S.; PEIXOTO, J. R. Melhoramento de plantas: variabilidade genética, ferramentas e mercado. Proimpress, Brasília, DF, Sociedade Brasileira de Melhoramento de Plantas, 108 p., 2018. Disponível em: $<$ https://www.infoteca.cnptia.embrapa.br/infoteca/bitstream/doc/1098740/1/Melhoram entodeplantas.pdf $>$.

FRANKHAM, R. Genetics and conservation biology. Comptes rendus Biologies, v. 326, p.22-29, 2003. Disponível em: <http://www.buffalofieldcampaign.org/archives/habitat/documents2/Frankham_Geneti cs_and_conservation.pdf>.

IBRAHIM, A. A.; MOHAMMAD, A.; BAKIRHASEEB, A. K.; FARHAN, A. H.; HOMAIDAN, A. A. et al. A Brief Review of Molecular Techniques to Assess Plant Diversity. International Journal of Molecular Sciences, v.11, n. 5, p. 2079-2096, 2010. Disponível em: <https://www.ncbi.nlm.nih.gov/pmc/articles/PMC2885095/pdf/ijms-1102079.pdf>. doi: <10.3390/ijms11052079>.

LIANG, Y.; ZHANG, Y.; WANG, G.; LI, Y., HUANG, W. Pendulifla worosin, a Diterpenoid from Croton crassifolius, Exerts Anti-Angiogenic Effect via VEGF Receptor-2 Signaling Pathway. Molecules, v.22, n.1, 2017. Disponível em: https://www.mdpi.com/1420-3049/22/1/126/htm.

doi:

https://dx.doi.org/10.3390/molecules22010126

MONDINI, L.; NOORANI, A.; PAGNOTTA, M. A. Assessing plant genetic diversity by molecular tools. Diversity, v.1, n.1, p.19-35, 2009. Disponível em: $<$ https://www.researchgate.net/publication/38104749_Assessing_Plant_Genetic_Div ersity_by_Molecular_Tools>.doi: 10.3390/d1010019.

NADEEM, M. A., NAWAZ, M. A., SHAHID, M. Q., DOĞAN, Y., COMERTPAY, G. et al. DNA molecular markers in plant breeding: current status and recent advancements in genomic selection and genome 
editing. Biotechnology \& Biotechnological Equipment, v. 32, n.2, p. 261-285, 2018. doi: https://doi.org/10.1080/13102818.2017.1400401

NASS, L. L. Pré-melhoramento vegetal. In Pré-melhoramento de Plantas. Estado da Arte e Experiências de Sucesso. In: LOPES, M. A.; FÁVERO, A. P.; FERREIRA, M. A. J. F.; FALEIRO, F. G.; FOLLE, S. M.; GUIMARÃES, E. P. Eds. Embrapa Informações Tecnológicas: Brasília, Brasil, p. 23-38, 2011.

NOGUEIRA, L. DE. M.; DA SILVA, M. R.; DOS SANTOS, S. M.; DE ALBUQUERQUE, J. F.; FERRAZ, I. C. et al. Antinociceptive Effect of the Essential Oil Obtained from the Leaves of Croton cordiifolius Baill. (Euphorbiaceae) in Mice. Evidence-Based Complementary and Alternative Medicine, v. 2015, p.1-7, 2015. doi: http://dx.doi.org/10.1155/2015/620865

OLIVEIRA, T. G.; PEREIRA, M. A. S.; COPPEDE, J. S.; FRANÇA, S. D. E. C.; MING, L. C. et al. Genetic diversity analysis of Croton antisyphiliticus Mart. using AFLP molecular markers. Genetics and Molecular Research, v.15, n.1, p.1-8, 2016. Disponível em: <http://www.funpecrp.com.br/gmr/year2016/vol151/pdf/gmr7461.pdf>. doi: http://dx.doi.org/10.4238/gmr.15017461

OSATHANUNKUL, M.; SUWANNAPOOM, C.; OUNJAI, S.; RORA, J. A.; MADESIS, $P$. et al. Refining DNA Barcoding Coupled High Resolution Melting for Discrimination of 12 Closely Related Croton Species. PLoS ONE, v.10, v.9, p.1-14, 2015. doi: https://doi.org/10.1371/journal.pone.0138888. Disponível em: https://journals.plos.org/plosone/article?id=10.1371/journal.pone.0138888

POLLITO, P. A. Z.; TOMAZELLO, M.; TAKASHIBA, H. E. Contribuição ao conhecimento do status de conservação das espécies do gênero Croton L. (Euphorbiaceae) no Brasil. Natureza e Conservação, v.2, p.42-55, 2004. Disponível em:

<https://www.researchgate.net/publication/265908136_Contribuicao_ao_conhecimen to_do_status_de_conservacao_das_especies_do_genero_Croton_L_Euphorbiaceae _no_Brasil>.

HAYWARD, A. C.; TOLLENAERE, R.; DALTON-MORGAN, J.; BATLEY, J. Molecular marker applications in plants. Methods in Molecular Biology, v.1245, p.13-27, 2015. doi: https://doi.org/10.1007/978-1-4939-1966-6_2

RIINA, R.; BERRY, P. E.; VAN EE, B. Molecular phylogenetics of the Dragon"s blood Croton section Cyclostigma (Euphorbiaceae): a polyphyletic assemblage unraveled. Systematic Botany, v. 34, p.360-374, 2009. Disponivel em: https://www.researchgate.net/publication/280988419_Molecular_Phylogenetics_of_th e_Dragon's_Blood_Croton_Section_Cyclostigma_Euphorbiaceae_A_Polypnyletic_As semblage_Unraveled. doi: 10.1600/036364409788606415

RIINA, R.; VAN Ee, B.; WIEDENHOEFT, A. C.; CARDOZO, A.; BERRY, P. E. Sectional rearrangement of arborescent clades of Croton (Euphorbiaceae) in South America: evolution of arillate seeds and a new species, Croton domatifer. Taxon, v.59, p.1147-1160, 2010. doi: 10.2307/20773984 
RIINA, R.; CARNEIRO-TORRES, D.; PEIRSON, J. A., BERRY, P. E., CORDEIRO, I. Further support for the Crotoneae phylogeny: a new species of Brasiliocroton (Euphorbiaceae) based on morphological, geographical, and molecular evidence. Systematic Botany, v.39, p.227-234, $2014 . \quad$ doi: https://doi.org/10.1600/036364414X678170

ROCHA, T. O.; FREITAS, J. S.; SANTOS, E. S. L.; SCALDAFERRI, M. M.; GONÇALVES, C. et al. Estimate of diversity and structure genetic in cassutinga (Croton heliotropiifolius) based on molecular markers. African Journal of Biotechnology, v.15, p.518-523, 2016. doi: https://doi.org/10.5897/AJB2015.15009

SCALDAFERRI, M. M.; FREITAS, J. S.; VIEIRA, J. G. P.; GONÇALVES, Z. S.; SOUZA, A. M. et al. Comparison of methods for estimates of molecular genetic diversity in genus Croton: influence of coefficients, clustering strategies and data projection. Genetics and Molecular Research, v.13, n.3, p. 5566-5573, 2014. Disponível em: https://pdfs.semanticscholar.org/ff57/5b8403478725bfc3a47d962dabfb2b1774b2.pdf. doi: http://dx.doi.org/10.4238/2014.July.25.11

SILVA, T. S. S.; FREITAS, J. S.; SANTOS, E. S. L.; CARDOSO, T. S.; CERQUEIRASILVA, C. B. Caracterização e seleção de marcadores moleculares em Croton linearifolius Mull. Arg. como subsídio para estudos genéticos. Multi-Science Journal, v. 1, n. 10, p. 4-8, 2018. Disponível em:

https://www.ifgoiano.edu.br/periodicos/index.php/multiscience/article/view/354/395

SILVA, T. S. S.; FREITAS, J. S.; SANTOS, E. S. L.; CERQUEIRA-SILVA, C. B. Diversity and genetic structure in natural populations of Croton linearifolius (Euphorbiaceae) based on molecular markers. African Journal of Biotechnology, no prelo.

SILVESTRINI, M.; PINTO-MAGLIO, C. A. F.; ZUCCHI, M. I.; SANTOS, F. A. M. Cytogenetics and characterization of microsatellite loci for a South American pioneer tree species, Croton floribundus. Genome, v.56, p.743-751, 2013. doi: https://doi.org/10.1139/gen-2013-0159.

SILVESTRINI, M.; MCCAULEY, D. E.; ZUCCHI, M. I.; SANTOS, F. A. M. How do gap dynamics and colonization of a human disturbed area affect genetic diversity and structure of a pioneer tropical tree species?. Forest Ecology and Management, v.344, p.38-52, 2015. doi: http://dx.doi.org/10.1016/j.foreco.2015.01.026

SOUZA, R. F.; ZIROLDO, B. D.; ROSSETTO, E. F.; CAVALHEIRO, A. L.; TOREZAN, J. M. D. et al. The use of genetic structure as a guide for seed gathering for forest restoration. Revista brasileira de Biociências, v.10, n.3, p.309-313, 2012. Disponível em: <http://www.ufrgs.br/seerbio/ojs/index.php/rbb/article/view/2039>.

TURChETTO-ZOLET, A. C.; TURChETto, C.; ZANELlA, C. M.; PASSAIA, G. (organizadores). Marcadores Moleculares na Era genômica: Metodologias e Aplicações. Ribeirão Preto: Sociedade Brasileira de Genética, 181

p., $2017 . \quad$ Disponível em: 
https://www.sbg.org.br/sites/default/files/e_book_marcadores_moleculares_sbg_201 7_final.pdf

VAN EE, B. W.; JELINSKI, N.; BERRY, P. E.; HIPP, A. L. (2006). Phylogeny and biogeography of Croton alabamensis (Euphorbiaceae), a rare shrub from Texas and Alabama, using DNA sequence and AFLP data. Molecular Ecology, 15: 2735-2751, 2006. doi: http://dx.doi.org/10.1111/j.1365-294X.2006.02970.x

VAN EE, B. W.; RIINA, R.; BERRY, P. E. A revised infrageneric classification and molecular phylogeny of New World Croton (Euphorbiaceae). Taxon, v.42, p.793-823, 2011.

https://www.researchgate.net/publication/233648946_A_revised_infrageneric_classifi cation_and_molecular_phylogeny_of_New_World_Croton_Euphorbiaceae. doi: $10.1002 / \operatorname{tax} .603013$

VAN EE, B. W.; BERRY, P. E. Croton section Pedicellati (Euphorbiaceae), a novel new world group, and a new subsectional classification of Croton section Lamprocroton. Systematic Botany, v.36, p.88-98, 2011. doi: https://doi.org/10.1600/036364411X553162

WEN-HUI, X.; WEI-YI, L.; QIAN, L. Chemical Constituents from Croton Species and Their Biological Activities. Molecules, v.23, n.9, p.1-38, 2018. doi: https://doi.org/10.3390/molecules23092333 